\title{
Do we have enough ophthalmologists to manage vision-threatening diabetic retinopathy? A global perspective
}

\author{
Zhen Ling Teo ${ }^{1} \cdot$ Yih-Chung Tham ${ }^{1,2} \cdot$ Marco Yu $\mathbb{1}^{1} \cdot$ Ching-Yu Cheng $\mathbb{D}^{1,2,3} \cdot$ Tien Yin Wong ${ }^{1,2,3} \cdot$ \\ Charumathi Sabanayagam (10) ${ }^{1,2}$
}

Received: 4 December 2019 / Accepted: 10 December 2019 / Published online: 28 January 2020

(c) The Author(s), under exclusive licence to The Royal College of Ophthalmologists 2020

\begin{abstract}
We aimed to estimate the supply of ophthalmologists in relation to the global and regional burden of vision-threatening diabetic retinopathy (VTDR). Diabetes mellitus (DM) population data from seven world regions were obtained from the International Diabetes Federation Atlas 2017. A systematic review was performed to include population-, community-based studies that reported country-specific VTDR prevalence. Random effect meta-analysis was then performed to estimate global and regional VTDR prevalence. VTDR prevalence estimates coupled with DM population data were then used to estimate the number of VTDR cases. Global and regional number of ophthalmologists were derived from the International Council of Ophthalmology Report 2015. Fifty studies (17 from Western Pacific [WP], nine North America and Caribbean [NAC], nine Middle East and North Africa [MENA], five Europe, eight South East Asia [SEA], one South and Central America [SACA] and one from Africa) were included. Global VTDR prevalence was 7.26\% (95\% CI, 6.18-8.32\%). Regional VTDR prevalence was $14.35 \%$ in Africa, $11.21 \%$ in MENA, $10.00 \%$ in NAC, $6.32 \%$ in Europe, $6.22 \%$ in WP, $5.83 \%$ in SACA and $2.97 \%$ in SEA. Globally, there were 7.16 ophthalmologists per 1000 VTDR patients. Europe had the highest ophthalmologist per 1000 VTDR patient ratio at 18.03 followed by SACA (17.41), while NAC, MENA and Africa had the lowest at 4.90, 4.81 and 0.91 respectively. Across regions, the ophthalmologist densities ranged from 0.91 to 18.03 per 1000 VTDR patients, with NAC, MENA and Africa having less than 5 ophthalmologists per 1000 patients. These findings will aid global and regional policy planning and healthcare resource allocation for VTDR management.
\end{abstract}

\section{Introduction}

The International diabetes federation (IDF) estimated the global diabetic population to be 425 million in 2017 and is set to rise to 628.6 million by 2045 [1]. Diabetic retinopathy

These authors contributed equally: Zhen Ling Teo, Yih-Chung Tham

Supplementary information The online version of this article (https:// doi.org/10.1038/s41433-020-0776-5) contains supplementary material, which is available to authorized users.

Charumathi Sabanayagam

charumathi.sabanayagam@seri.com.sg

1 Singapore Eye Research Institute, Singapore National Eye Centre, The Academia, 20 College Road, Discovery Tower Level 6, Singapore 169856, Singapore

2 Duke-NUS Medical School, Singapore, Singapore

3 Yong Loo Lin School of Medicine, National University of Singapore, Singapore, Singapore
(DR) is a common diabetic complication [2] and the leading cause of preventable blindness in the adult working population [3]. A previous meta-analysis reported a high global DR prevalence of $34.6 \%$ [4]. The prevalence of visionthreatening DR (VTDR), a more severe form of DR, was estimated to be $10.2 \%$ [4]. With the burgeoning DR population, the corresponding demand for VTDR treatment will also increase, and thus a further strain on ophthalmologist manpower.

As shown in developed countries such as Singapore, Portland and United Kingdom, mild or moderate DR can be effectively managed by trained primary care physicians, optometrists and nurses [5-9]. However, management of VTDR requires the expertise and skills of trained ophthalmologists or retinal specialists [10]. Key sight-saving treatments such as focal lasers, panretinal photocoagulation (PRP) [10], intravitreal anti vascular endothelial growth factor treatment (IVT) [11-13] and vitreoretinal surgery can only be performed by trained ophthalmologists or retinal specialists. Hence, ophthalmologists are the key manpower 
resource for the management of VTDR. The World Health Organisation's global action plan for Vision 2020 has identified the development of human resources for eye health as a key pillar for the prevention of avoidable blindness and visual impairment. It recommends that national programmes train and maintain an eye health workforce proportionate to the needs of the population [14]. Hence, estimation on ophthalmologist manpower in this regard will provide further insights on each regional healthcare systems' current capability in handling the burden of VTDR.

There is currently no study that has evaluated the ratio of ophthalmologist workforce to VTDR patients at either country or regional level. Hence, there is a pressing need to evaluate this aspect to better determine if we have sufficient ophthalmologists to meet the growing burden of VTDR. To address this limitation, we aimed to provide estimates on the number of ophthalmologists available per 1000 VTDR population (ophthalmologist density) at global and regional levels.

\section{Methods}

\section{Search strategy for diabetes mellitus (DM) and VTDR prevalence}

Data from the IDF Atlas 8th edition 2017 provided countryspecific estimates on the number of adults with diabetes in millions, for 194 countries. We grouped these countries according to the seven IDF world regions. To obtain VTDR prevalence, a literature search of all published papers using international electronic databases, PubMed, Web of Science, Open Grey, was conducted. Key words included "diabetic retinopathy", "prevalence", "global prevalence" and specific country names. We included publications up to 13 August 2019. Further literature search consisted of reviewing reference lists of relevant articles.

\section{Inclusion and exclusion criteria}

We included population-based studies, community-based studies and national surveys. We manually reviewed the full text articles and included studies that provided original data for the number of DM and number of DR cases. Published papers in English were included for evaluation. Articles were reviewed independently by two reviewers (ZLT and YCT) and selected for inclusion. Any disagreement was resolved by a third reviewer who subsequently reviewed the list of selected articles (CS). Only participants with diabetes were included for assessing the prevalence of DR and VTDR. We included studies that had DR diagnosis made from colour fundus photographs or dilated fundal examinations by an ophthalmologist.
Studies that were duplicates or that had utilised data of a previously studied population were excluded. We excluded papers that solely studied $\mathrm{T} 1$ diabetic populations, studies with unclear study design, studies that did not have a clear definition of DM for patient selection and studies that had a small study sample size of less than 150 DM participants.

\section{Definition of DM, DR and VTDR}

A variety of modalities was used to identify patients with DM depending on the study and country. Modalities used included fasting blood glucose $\geq 7 \mathrm{mmol} / \mathrm{L}$, random blood glucose $>11.1 \mathrm{mmol} / \mathrm{L}$, oral glucose tolerance test $\geq 11.1 \mathrm{mmol} / \mathrm{L}$, glycated haemoglobin $(\mathrm{A} 1 \mathrm{c}) \geq 6.5 \%$, selfreporting of physician diagnosed DM, existing DM treatment and medical records.

Majority of studies defined DR by the presence of retinal haemorrhages, microaneurysms, cotton wool spots and/or PRP laser scars according to the Early treatment diabetic retinopathy study (ETDRS) classification [15] or American Association of Ophthalmology (AAO) International clinical DR disease severity scale [16]. Diabetic macular oedema (DMO) was classified with the International Diabetic Macular Clinical Edema Severity Scale [16]. VTDR was defined as the presence of severe NPDR, proliferative DR (PDR) and/or clinically significant macular oedema according to the Eye Diseases Prevalence Research Group definition [17].

\section{Obtaining VTDR population data}

Prevalence estimates from all included studies were metaanalysed to obtain global and regional VTDR prevalence estimates. The regional prevalence estimate was then applied to the DM population data of individual countries of that region to obtain country- and region-specific VTDR population estimates.

The regional DM population data provided by IDF Atlas 2017 were estimated and thus $95 \%$ confidence interval was calculated to reflect the range in which the "true number of adults with DM" may lie. Regional VTDR prevalence estimates was thus also applied to the upper limit and lower limits of DM population data for each region to obtain the upper and lower limit of the estimated VTDR population size.

\section{Identifying population of active ophthalmologists}

A 2015 study by the International Council of Ophthalmology (ICO) provided the number of active ophthalmologists of 194 countries [18]. These countries were sorted according to IDF regions. The ophthalmologist density is defined as the number of available ophthalmologists per 1000 
VTDR patients. The ophthalmologist density was obtained by dividing the ophthalmologist population by the mean, upper and lower limit of estimated VTDR population (in 1000 s) globally and for each region.

\section{Statistical analysis}

VTDR prevalence was calculated per study for total population and geographic-specific subgroups. Random-effects meta-analysis was performed by the inverse variance method. Test for heterogeneity (I-squared index) was performed to determine any significant difference in prevalence estimates across studies. All statistical analyses were performed using the statistical software $\mathrm{R}$ (version 3.4.4; $\mathrm{R}$ Foundation, Vienna, Austria). $P$ value $<0.05$ was considered statistically significant.

\section{Results}

Figure 1 shows the article selection process for studies included in the final meta-analyses. In brief, a total of 3255 individual articles were identified with our initial strategy, out of which 76 articles were relevant to our review. After reviewing the full text of the selected articles, 26 articles that did not fulfil our inclusion criteria were excluded. In the end, we included 41,712 DM patients from 50 studies from 26 countries from 7 IDF world regions (refer to Supplementary Fig. 1 for study details). Meta-analysis of the 50 studies gave a global VTDR prevalence estimate of $7.26 \%$ (95\% CI 6.18-8.33\%) as shown in Fig. 2 with an I-squared index of $97 \%$ showing heterogeneity. Africa had the highest regional VTDR prevalence at $14.36 \%$ and South East Asia (SEA) had the lowest regional VTDR prevalence at $2.97 \%$. The regional VTDR prevalence of other regions are as follows: Middle East and North Africa (MENA) $11.21 \%$, North America and Caribbean (NAC) $10.00 \%$, Europe 6.32\%, Western Pacific (WP) 6.22\% and South and Central America (SACA) 5.83\%. Table 1 shows the estimated number of adults with diabetes and VTDR by region. The number of adults with diabetes and VTDR ranged from 2.2 million in Africa to 10.47 million in the WP region.

Table 2 shows the ophthalmologist density by the mean, upper and lower limit of the VTDR population. Ophthalmologist density at the global level was 7.16; regionally, Europe had the highest density (18.03) and Africa had the lowest (0.91). In the worse-case scenario where the upper limit of VTDR population estimate was used, the lowest ophthalmologist density was seen in Africa at 0.49 , while SACA had the highest with 14.01. In the best-case scenario, the highest ophthalmologist density was found in Europe at 22.82 and the lowest in Africa at 1.46. This varying ophthalmologist densities across the world regions is also

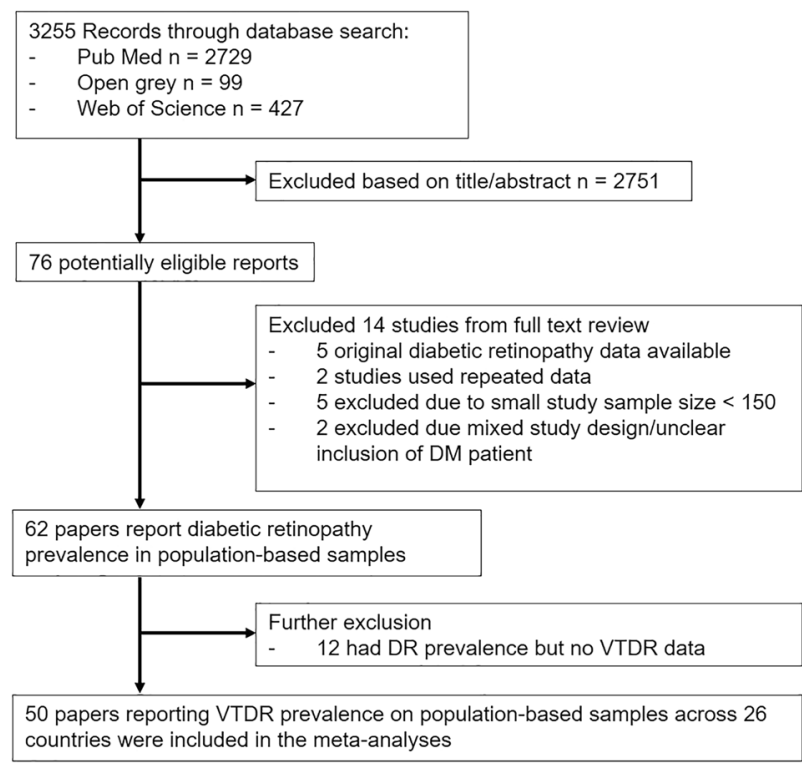

Fig. 1 Summary of article selection process.

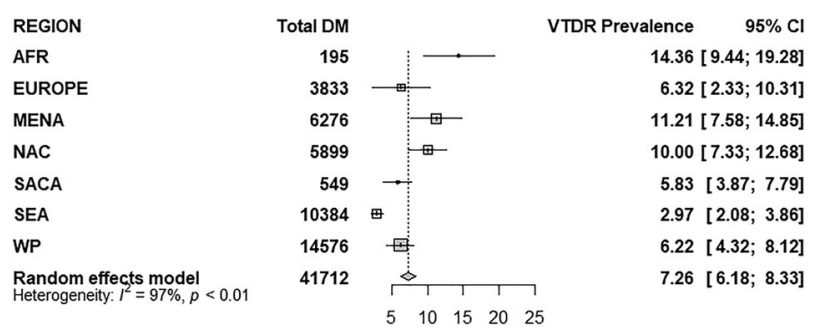

Fig. 2 VTDR prevalence estimate amongst DM population for each world region and pooled global VTDR estimate derived from meta-analysis.

illustrated in Fig. 3 with "hotter" denser colours representing higher density of ophthalmologist per patient population and lighter colours representing a lower density.

The top three regions with highest VTDR prevalence were Africa followed by MENA and NAC as mentioned above. Correspondingly, these three regions also had the lowest ophthalmologist densities: Africa (0.91), MENA (4.81) and NAC (4.90). The ophthalmologist densities were low in countries with high VTDR prevalence. The top three regions with highest absolute number of adults with VTDR were WP, followed by NAC and then MENA (Table 1). The top three regions with highest absolute number of ophthalmologists was found in Europe followed by WP and then SACA (Table 2). SACA had the smallest VTDR population but had the second highest absolute number of ophthalmologists.

In addition to regional information, we further illustrated country-specific ophthalmologist density (Supplementary Fig. 2 and Supplementary Table 1). The country with the highest ophthalmologist density is Greece with 45.58 active ophthalmologists available per 1000 VTDR patients. The second and third highest ophthalmologist density was found 
Table 1 Estimated DM and VTDR population by regions.

\begin{tabular}{|c|c|c|c|c|c|c|c|}
\hline \multirow[t]{2}{*}{ Region } & \multicolumn{3}{|c|}{$\begin{array}{l}\text { Adults with diabetes mellitus, in } \\
\text { millions }\end{array}$} & \multirow[t]{2}{*}{$\begin{array}{l}\text { VTDR prevalence } \\
\text { estimate }(\%)\end{array}$} & \multicolumn{3}{|c|}{ Adults with VTDR, in millions } \\
\hline & Mean & $\begin{array}{l}\text { Lower } \\
\text { limit }^{\mathrm{a}}\end{array}$ & $\begin{array}{l}\text { Upper } \\
\text { limit }^{\mathrm{a}}\end{array}$ & & Mean & $\begin{array}{l}\text { Lower } \\
\text { limit }^{\mathrm{a}}\end{array}$ & $\begin{array}{l}\text { Upper } \\
\text { limit }^{\mathrm{a}}\end{array}$ \\
\hline AFR & 15.34 & 9.59 & 28.44 & 14.36 & 2.20 & 1.38 & 4.08 \\
\hline EUR & 65.47 & 51.74 & 94.23 & 6.32 & 4.14 & 3.27 & 5.96 \\
\hline MENA & 39.70 & 27.76 & 52.99 & 11.21 & 4.45 & 3.11 & 5.94 \\
\hline NAC & 49.93 & 41.68 & 55.91 & 10.00 & 4.99 & 4.17 & 5.59 \\
\hline SACA & 27.57 & 22.74 & 34.26 & 5.83 & 1.61 & 1.33 & 2.00 \\
\hline SEA & 83.62 & 63.57 & 107.44 & 2.97 & 2.48 & 1.89 & 3.19 \\
\hline WP & 168.26 & 149.52 & 210.66 & 6.22 & 10.47 & 9.30 & 13.10 \\
\hline Global & 449.89 & 366.59 & 583.93 & 7.26 & 32.66 & 26.61 & 42.39 \\
\hline
\end{tabular}

AFR Africa, EUR Europe, MENA Middle East and North Africa, NAC North America and Caribbean, SACA South and Central America, SEA South East Asia, WP Western Pacific.

${ }^{a}$ Based on $95 \%$ confidence interval of estimated DM population per region provided by IDF Atlas 2017 .
Table 2 Ophthalmologist densities of each regional VTDR population.

\begin{tabular}{llrcc}
\hline Region & $\begin{array}{l}\text { Number of } \\
\text { ophthalmologists }\end{array}$ & \multicolumn{2}{l}{$\begin{array}{l}\text { Number of ophthalmologists per 1000 } \\
\text { VTDR population }\end{array}$} \\
\cline { 3 - 5 } & & $\begin{array}{c}\text { Mean } \\
\text { Lewer limit } \\
\text { of VTDR } \\
\text { population }\end{array}$ & $\begin{array}{l}\text { Upper limit of } \\
\text { VTDR } \\
\text { population }\end{array}$ \\
\hline AFR & 2007 & 0.91 & 1.46 & 0.49 \\
EUR & 74,615 & 18.03 & 22.82 & 12.53 \\
MENA & 21,419 & 4.81 & 6.88 & 3.61 \\
NAC & 24,444 & 4.90 & 5.86 & 4.37 \\
SACA & 27,987 & 17.41 & 21.11 & 14.01 \\
SEA & 18,403 & 7.41 & 9.75 & 5.77 \\
WP & 64,969 & 6.21 & 6.99 & 4.96 \\
Global & 233,844 & 7.16 & 8.79 & 5.52 \\
\hline
\end{tabular}

AFR Africa, EUR Europe, MENA Middle East and North Africa, NAC North America and Caribbean, SAC South and Central America, SEA South East Asia, WP Western Pacific.

${ }^{a}$ Ratio for each region is obtained by dividing the number of ophthalmologists by VTDR population number listed in Table 1.

in Lithuania (42.84) and Argentina (42.55), respectively. Five countries (Cook Islands, Federated states of Micronesia, Nauru, Niue, Tuvalu) had reportedly no currently available ophthalmologists and thus yielding zero density value. A comparison of ophthalmologist densities between the three highest and three lowest income countries are shown in Table 3. The ophthalmologist density is 0.71 in the lowest income country (Burundi) and 28.77 in the highest income country (Luxembourg).

\section{Discussion and conclusion}

In this study, we estimated VTDR prevalence, VTDR population size and its corresponding ophthalmologist density globally and regionally. We focused on ophthalmologist to VTDR patient ratio as VTDR management requires ophthalmologists, whereas mild and moderate NPDR can be managed by primary care physicians and other allied health professionals [5-9]. Globally, ophthalmologist density is low, and is especially notable in regions such as Africa, MENA and NAC, with the lowest in Africa (0.91). This is the first study that attempted to estimate the sufficiency of ophthalmologists for VTDR management. These findings will assist in improving the design of global and regional action plans to better tackle VTDR.

This study found that more than half the countries had a low ophthalmologist density of less than 5.41. The ophthalmologist density can also vary greatly within a region such as in Europe ranging from 4.3 (Tajikistan) to 45.6 (Greece) and SACA ranging from 5.3 (Nicaragua) to 42.6 (Argentina). However, it should also be noted that due to the varying healthcare operating models adopted by different countries, the ideal ratio of ophthalmologist to VTDR patients required to cope with demand will naturally differ. At the same time, the ICO guidelines recommend resource based DR care such as lower DR screening frequency in low or intermediate resource settings [19]. Hence, manpower requirements may differ based on resource setting and the provision of region and country-specific ophthalmologist densities can assist individual countries and region in manpower planning and training.

It is interesting to note that NAC had the third lowest ophthalmologist to 1000 VTDR ratio (4.90) despite it comprising of developed countries including the United States of America and Canada. Although NAC had a fair absolute number of ophthalmologists $(24,444)$, it had the third highest VTDR prevalence and the second highest estimated absolute number of adults with VTDR at 5.0 million (95\% CI 4.17-5.59) (Table 1). This shows that while we can attempt to increase ophthalmologist 


\section{Ophthalmologist densities per 1000 VTDR population across world regions}
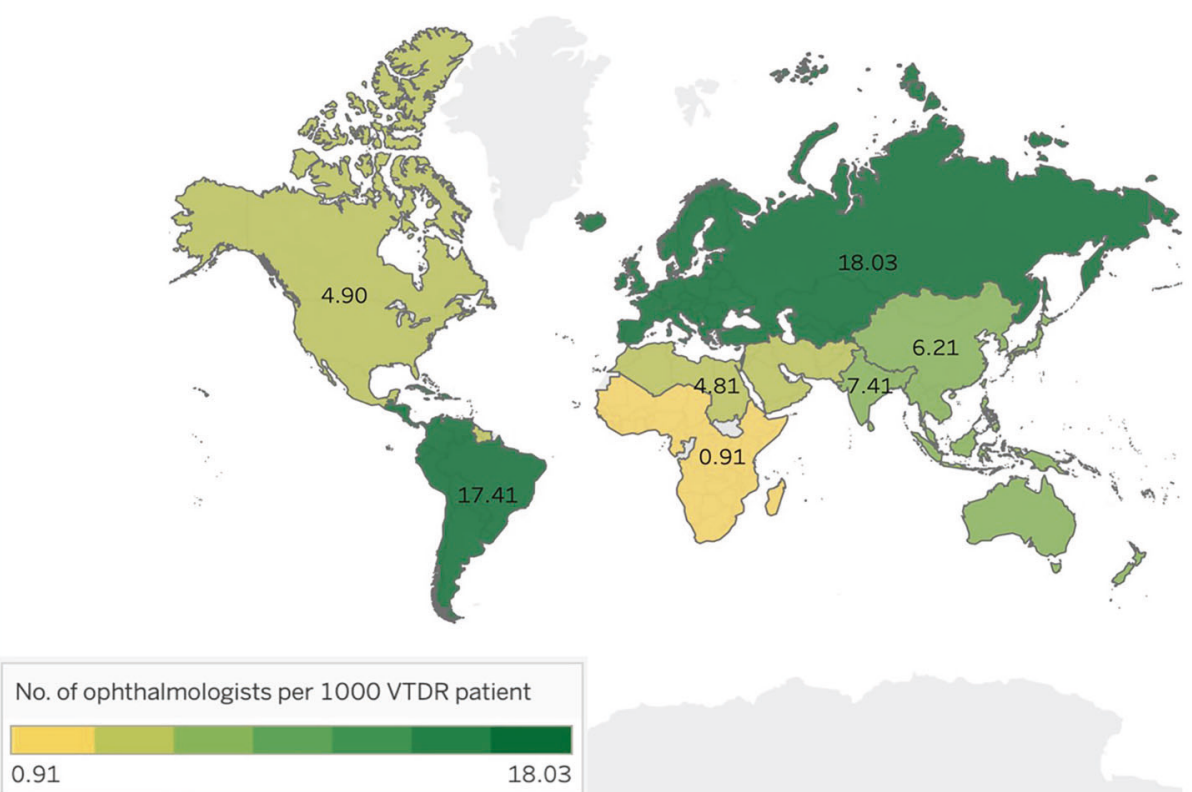

Fig. 3 Ophthalmologist densities across the world by regions.

Table 3 Comparison of VTDR prevalence and ophthalmologist densities between the highest and lowest income countries based on World Bank 2017 rankings.

\begin{tabular}{|c|c|c|c|c|}
\hline $\begin{array}{l}\text { Highest income } \\
\text { Top three countries }\end{array}$ & Region & DM prevalence $(\%)$ & $\begin{array}{l}\text { Regional VTDR } \\
\text { prevalence estimate }\end{array}$ & $\begin{array}{l}\text { Number of ophthalmologists } \\
\text { per } 1000 \text { VTDR patient }\end{array}$ \\
\hline Luxembourg & EUR & 5.89 & 0.0632 & 28.77 \\
\hline Switzerland & & 7.89 & & 23.98 \\
\hline Norway & & 8.11 & & 19.71 \\
\hline $\begin{array}{l}\text { Lowest income } \\
\text { Bottom three countries }\end{array}$ & Region & DM prevalence $(\%)$ & $\begin{array}{l}\text { Regional VTDR } \\
\text { prevalence estimate }\end{array}$ & $\begin{array}{l}\text { Number of ophthalmologists } \\
\text { per } 1000 \text { VTDR patient }\end{array}$ \\
\hline Burundi & AFR & 2.36 & 0.1436 & 0.71 \\
\hline Malawi & & 2.26 & & 0.31 \\
\hline Nigeria & & 1.81 & & 2.20 \\
\hline
\end{tabular}

manpower, we also need concurrent measures to reduce VTDR rates. Measures to prevent blindness from DR include effective primary and secondary prevention such as early detection by screening, effective DM management and compliance to recommended DR guidelines [20]. It has been shown that patients who receive consistent care according to guidelines have lower rates of low vision and blindness [21]. The application of deep learning to assist DR screening will potentially play a key role in mass DR screening in years to come [20, 22, 23].

There was also a disparity in resources and DM burden across high- and low-income countries (Table 3). According to World bank data 2017 the top three countries with the highest gross national income were found in Europe: Luxembourg, Switzerland and Norway. The three countries with the lowest gross national income were found in Africa:
Burundi, Malawi and Nigeria. Higher income countries correspondingly had significantly higher ophthalmologist densities despite low- and middle-income countries carrying $75 \%$ of the burden of DM [24].

In view of high VTDR numbers especially in lower income countries, VTDR treatment should be cost effective. Studies by the Diabetic Retinopathy Clinical Research Network showed that visual outcomes and 2-year PDR status are comparable between IVT Ranibizumab and PRP for PDR (Protocol S) [25]. However, IVT Ranibizumab is currently not cost effective for patients with PDR without vision-threatening CSME [26, 27]. The Protocol V trial also showed that there is no significant difference in 2-year vision loss between IVT aflibercept, laser photocoagulation and observation for patients with centre-involving DMO with good visual acuity [28]. This knowledge will allow 
ophthalmologists to practice more cost-effective VTDR management.

\section{Strengths and limitations}

A strength of our study is the inclusion of studies from all seven world regions in the meta-analysis to obtain regional and country level VTDR population estimates matched to ophthalmologist data. This allows for national planning of healthcare and manpower. The provision of prevalence estimates with a $95 \%$ confidence interval range in which the true VTDR population may lie also allows for regions and countries to estimate their manpower needs while considering the spectrum of possible scenarios.

Our study has several limitations. The pooling of multiple studies with heterogenous research designs can affect the accuracy of our VTDR prevalence estimate. In addition, for Africa and SACA, only one suitable study was found in each region for the estimation of regional VTDR prevalence. Regional VTDR estimate is applied onto DM population under the assumption that countries in the same IDF region may have the closest VTDR prevalence resulting in a discrepancy at the country level. While majority of studies used either the ETDRS or AAO International Clinical Diabetic Retinopathy Severity Scale classification, seven studies used the Scottish Diabetic Retinopathy Grading Scheme, which did not include severe NPDR in VTDR. This may underestimate the VTDR prevalence and represents a further strain on ophthalmologist manpower.

In reality, the ophthalmologist density is likely lower with a large number of undiagnosed DM patients that is unaccounted for. In 2017, it is estimated globally that 224 million individuals aged 18-99 years with DM are unaware of their condition [1]. With greater education efforts and more screening programmes, the number of VTDR patients requiring treatment will rise, adding further strain to existing manpower resources. At present, studies have shown that many countries are unable to achieve the human resource targets of the Vision 2020 Global eye health strategy aimed at reducing the prevalence of avoidable visual impairment by year 2020 [14].

In view of these limitations, future studies on VTDR especially in Africa and SACA are essential to allow for more robust estimates. Individual countries are also encouraged to engage in more studies to provide countryspecific VTDR prevalence and population data so as to allow for better planning of national healthcare policies aimed at saving vision.

In conclusion, there is a low ratio of ophthalmologist to VTDR patients globally, and a substantial disparity in ophthalmologist densities across different regions. This highlights the need to enhance the ophthalmology workforce and calls for new innovative strategies such as artificial intelligence for VTDR management. Findings in this study can aid in the planning of global, regional and country-specific healthcare strategies to prevent diabetes related vision loss.

Acknowledgements We also thank Ms. Riswana Banu Binte Mohamed Abdul, Singapore Eye Research Institute, Singapore for her help with the formatting of the manuscript.

\section{Compliance with ethical standards}

Conflict of interest The authors declare that they have no conflict of interest.

Publisher's note Springer Nature remains neutral with regard to jurisdictional claims in published maps and institutional affiliations.

\section{References}

1. Cho NH, Shaw JE, Karuranga S, Huang Y, da Rocha Fernandes JD, Ohlrogge AW, et al. IDF Diabetes Atlas: global estimates of diabetes prevalence for 2017 and projections for 2045. Diabetes Res Clin Pract. 2018;138:271-81.

2. Khalil H. Diabetes microvascular complications - a clinical update. Diabetes Metab Syndr. 2017;11(Suppl 1):S133-S9.

3. Cheung N, Mitchell P, Wong TY. Diabetic retinopathy. Lancet. 2010;376:124-36.

4. Yau JW, Rogers SL, Kawasaki R, Lamoureux EL, Kowalski JW, Bek T, et al. Global prevalence and major risk factors of diabetic retinopathy. Diabetes Care. 2012;35:556-64.

5. Nguyen HV, Tan GS, Tapp RJ, Mital S, Ting DS, Wong HT, et al. Cost-effectiveness of a National Telemedicine Diabetic Retinopathy Screening Program in Singapore. Ophthalmology. 2016; 123:2571-80.

6. Bhargava M, Cheung CY, Sabanayagam C, Kawasaki R, Harper CA, Lamoureux EL, et al. Accuracy of diabetic retinopathy screening by trained non-physician graders using non-mydriatic fundus camera. Singap Med J. 2012;53:715-9.

7. Mansberger SL, Sheppler C, Barker G, Gardiner SK, Demirel S, Wooten $\mathrm{K}$, et al. Long-term comparative effectiveness of telemedicine in providing diabetic retinopathy screening examinations: a randomized clinical trial. JAMA Ophthalmol. 2015;133:518-25.

8. Scanlon PH. The English National Screening Programme for diabetic retinopathy 2003-2016. Acta Diabetol. 2017;54:515-25.

9. Olson JA, Strachan FM, Hipwell JH, Goatman KA, McHardy KC, Forrester JV, et al. A comparative evaluation of digital imaging, retinal photography and optometrist examination in screening for diabetic retinopathy. Diabet Med. 2003;20:528-34.

10. Indications for photocoagulation treatment of diabetic retinopathy: diabetic retinopathy study report no. 14. The Diabetic Retinopathy Study Research Group. Int Ophthalmol Clin. 1987;27:239-53.

11. Sun JK, Glassman AR, Beaulieu WT, Stockdale CR, Bressler $\mathrm{NM}$, Flaxel C, et al. Rationale and application of the protocol S Anti-vascular endothelial growth factor algorithm for proliferative diabetic retinopathy. Ophthalmology. 2019;126:87-95.

12. Wells JA, Glassman AR, Ayala AR, Jampol LM, Aiello LP. Diabetic Retinopathy Clinical Research $\mathrm{N}$ et al. Aflibercept, bevacizumab, or ranibizumab for diabetic macular edema. N. Engl J Med. 2015;372:1193-203.

13. Simunovic MP, Maberley DA. Anti-vascular endothelial growth factor therapy for proliferative diabetic retinopathy: a systematic review and meta-analysis. Retina. 2015;35:1931-42. 
14. Palmer JJ, Chinanayi F, Gilbert A, Pillay D, Fox S, Jaggernath J, et al. Mapping human resources for eye health in 21 countries of sub-Saharan Africa: current progress towards VISION 2020. Hum Resour Health. 2014;12:44.

15. Grading diabetic retinopathy from stereoscopic color fundus photographs--an extension of the modified Airlie House classification. ETDRS report number 10. Early Treatment Diabetic Retinopathy Study Research Group. Ophthalmology. 1991;98(5 Suppl):786-806.

16. Wilkinson CP, Ferris FL 3rd, Klein RE, Lee PP, Agardh CD, Davis $\mathrm{M}$, et al. Proposed international clinical diabetic retinopathy and diabetic macular edema disease severity scales. Ophthalmology. 2003;110:1677-82.

17. Kempen JH, O'Colmain BJ, Leske MC, Haffner SM, Klein R, Moss SE, et al. The prevalence of diabetic retinopathy among adults in the United States. Arch Ophthalmol. 2004;122:552-63.

18. Resnikoff S, Lansingh VC, Washburn L, Felch W, Gauthier TM, Taylor HR, et al. Estimated number of ophthalmologists worldwide (International Council of Ophthalmology update): will we meet the needs? Br J Ophthalmol. 2019. [Epub ahead of print].

19. Wong TY, Sun J, Kawasaki R, Ruamviboonsuk P, Gupta N, Lansingh VC, et al. Guidelines on diabetic eye care: The International Council of Ophthalmology recommendations for screening, follow-up, referral, and treatment based on resource settings. Ophthalmology. 2018;125:1608-22.

20. Wong TY, Sabanayagam C. Strategies to tackle the global burden of diabetic retinopathy: from epidemiology to artificial intelligence. Ophthalmologica. 2020;243:9-20.

21. Sloan FA, Grossman DS, Lee PP. Effects of receipt of guidelinerecommended care on onset of diabetic retinopathy and its progression. Ophthalmology. 2009;116:1515-21.e1-3.

22. Sayres R, Taly A, Rahimy E, Blumer K, Coz D, Hammel N, et al. Using a deep learning algorithm and integrated gradients explanation to assist grading for diabetic retinopathy. Ophthalmology. 2019;126:552-64.

23. Gulshan V, Peng L, Coram M, Stumpe MC, Wu D, Narayanaswamy $\mathrm{A}$, et al. Development and validation of a deep learning algorithm for detection of diabetic retinopathy in retinal fundus photographs. JAMA. 2016;316:2402-10.

24. Ogurtsova K, da Rocha Fernandes JD, Huang Y, Linnenkamp U, Guariguata L, Cho NH, et al. IDF Diabetes Atlas: global estimates for the prevalence of diabetes for 2015 and 2040. Diabetes Res Clin Pract. 2017;128:40-50.

25. Gross JG, Glassman AR, Jampol LM, Inusah S, Aiello LP. Writing Committee for the Diabetic Retinopathy Clinical Research N, et al. Panretinal photocoagulation vs intravitreous ranibizumab for proliferative diabetic retinopathy: a randomized clinical trial. JAMA. 2015;314:2137-46.

26. Hutton DW, Stein JD, Glassman AR, Bressler NM, Jampol LM, Sun JK, et al. Five-year cost-effectiveness of intravitreous ranibizumab therapy vs panretinal photocoagulation for treating proliferative diabetic retinopathy: a secondary analysis of a randomized clinical trial. JAMA Ophthalmol. 2019:1-9. [Epub ahead of print].

27. Hutton DW, Stein JD, Bressler NM, Jampol LM, Browning D, Glassman AR, et al. Cost-effectiveness of intravitreous ranibizumab compared with panretinal photocoagulation for proliferative diabetic retinopathy: secondary analysis from a diabetic retinopathy clinical research network randomized clinical trial. JAMA Ophthalmol. 2017;135:576-84.

28. Baker CW, Glassman AR, Beaulieu WT, Antoszyk AN, Browning DJ, Chalam KV, et al. Effect of initial management with aflibercept vs laser photocoagulation vs observation on vision loss among patients with diabetic macular edema involving the center of the macula and good visual acuity: a randomized clinical trial. JAMA. 2019;321:1880-94. 